\title{
El cambio de la práctica médica. Desafíos psicosociales para la profesión
}

\author{
Nina Horwitz $\mathbf{C}^{1}$. \\ The change in medical practice. \\ Psycho-social challenges for the \\ profession
}

This article analyzes important changes in medical practice, focusing in those most deeply perceived by a group of physicians from the Metropolitan Region of Santiago, included in a joint research conducted by the Departments of Psychiatry and Mental Health (East) of the Medical Faculty at the University of Chile and Public Health of the Catholic University of Santiago, during 2003. These are the perceived changes in the relationship between doctors and patients; increased limits in professional autonomy and the fragmentation of medical practice. Reflecting transformations in social relationships in general, they have added new stress and frustrations, as well as new opportunities and rewards to the medical profession. The perceptions identified are commented within the structural determinants of medical practice. These issues call for a refreshing discussion on the values supporting medical professionalism and the concept of profession in itself, in view of the challenges posed by the current social and cultural changes (Rev Méd Chile 2004; 132: 768-72).

(Key Words: Practice management, medical; Sociology, medical)

Recibido el 27 de enero, 2004. Aceptado en versión corregida el 12 de marzo, 2004.

${ }^{1}$ Socióloga, Departamento de Psiquiatría y Salud Mental, Campus Oriente y Departamento de Bioética y Humanidades Médicas, Facultad de Medicina, Universidad de Chile. Santiago de Chile.

L os variados y acelerados cambios en la organización social de la práctica médica, ocurridos en las últimas tres décadas invitan a un proceso de redefinición interna de la práctica profesional. Con ese objetivo resulta interesante revisar el concepto de profesión, ya que el impacto psicosocial de los cambios afecta las expectativas construidas culturalmente y una ética de las profesiones requiere recordar cuál es la naturaleza y el sentido de estas actividades sociales.

Este trabajo está basado en un análisis de los principales resultados del estudio Bienestar y satisfacción de los médicos en el ejencicio de su profesión en un sistema de salud en proceso de

Correspondencia a: Prof. Nina Horwitz C. Salvador 486, Providencia. Fono-Fax: 2748855. E-mail: nhorwitz@med.uchile.cl reforma»de los Departamentos de Psiquiatría y Salud Mental Oriente, de la Facultad de Medicina de la Universidad de Chile y de Salud Pública de la Pontificia Universidad Católica, durante el año 2003.

El estudio utilizó un método cualitativo, con una muestra de 44 médicos de distintas edades, especialidades y situaciones de práctica profesional (sectores público/académico; privado, urgencia y atención primaria/medicina familiar). Se aplicaron dos métodos de recolección de datos a distintos grupos de sujetos: un cuestionario autoadministrado y tres grupos focales, sobre la base de las mismas tres preguntas básicas siguientes:

1. ¿Cuáles son los cambios principales que los médicos perciben que se han producido en la práctica de la medicina durante el período en que han ejercido? 
2. ¿Cuál es la percepción del impacto que tales cambios han tenido sobre el grado de satisfacción con la práctica y la calidad de vida de los médicos?

3. ¿Cuáles han sido las principales estrategias de afrontamiento (coping) y de supervivencia que los médicos han aplicado frente a estos cambios?

Se realizó un análisis de contenido de los datos que fue sometido a una triangulación por el equipo interdisciplinario de investigación. Este análisis permitió identificar los factores principales y de mayor relevancia (de acuerdo a su frecuencia e intensidad).

Este artículo abordará la percepción de cambios en la relación social entre médicos y pacientes; en las limitaciones de la autonomía profesional y la segmentación de la profesión médica.

1. La percepción de cambios en la relación social entre médicos y pacientes. El más notorio de los cambios percibidos por los médicos, ha ocurrido en la interacción entre los médicos y los pacientes, atribuido al mayor acceso a la información sobre temas médicos por la población y a una actitud más afirmativa de parte de ésta. En muchos sujetos del estudio existe conciencia que este cambio es el reflejo de cambios mayores en la sociedad:

«on cambios en la sociedad chilena. De una sociedad paternal preocupada por la seguridad social donde la salud era parte de la seguridad social y buena educación (el gobierno educó a mis hijos); ahora no, la sociedad es cada vez más individualista. Se pasa a ser objeto de consumo que modifica la relación profesional médico paciente». (Médico, Grupo Focal III).

La responsabilidad de organizar la oferta de los servicios profesionales recae hoy progresivamente en organizaciones que racionalizan la demanda de los servicios profesionales. Sobre todo en el sector privado, se percibe que la relación con los pacientes se va desperfilando.

«o otro es cómo ha ido evolucionando la consulta médica, ha ido quedando atrás la consulta del médico, ahora hay, cada vez más, centros de mega atención, donde el paciente resuelve todos sus problemas». (Médico, Grupo Focal II).

«os médicos hemos visto cómo disminuye el número de pacientes que pagan directamente de su bolsillo y cómo aumentan los sistemas de ejercicio en grupo, sea a través de ISAPRES, de seguros u otras modalidades de financiamiento». (Encuesta I).

El paciente parece sentir menos trabas para cuestionar directa o indirectamente la conducta profesional médica. Ahora la actitud es más crítica, aparece como más recelosa y de menor asimetría, produciéndose diversas formas de desafío de la autoridad médica, en un continuo desde las formas más silenciosas a las más estridentes, litigantes y violentas, dando pie al surgimiento de la llamada medicina defensiva:

«mpieza la medicina por el miedo,... no basada en la evidencia sino basada en el miedo, miedo a que todo lo del paciente les caiga encima y así existen muchas cosas que se hacen demás y otras de menos». (Médico, Grupo Focal III).

Más allá de la medicalización de la sociedad, entre las interpretaciones recientes de los cambios en la relación médico paciente, ciertamente el llamado «clima de época»que empezó a ser descrito en las postrimerías del siglo veinte, se ubica entre las principales. La modemidad tardía» se caracteriza por un creciente ejencicio reflexivo ${ }^{1}$. Es decir, una proporción mayoritaria de las dimensiones de la vida social y de las relaciones materiales con el ambiente natural se hacen susceptibles a una constante revisión, a la luz de la renovación del conocimiento y de la información. La mentalidad moderna institucionaliza la duda radical, al insistir que todo conocimiento es tentativo y perfectible. Anthony Giddens ${ }^{2}$ afirma, a propósito de la globalización, que nuestra relación con la ciencia y la tecnología es hoy muy diferente a la que existió en épocas anteriores, en que la ciencia funcionaba como otra forma de tradición, en el sentido que la gente la respetaba, pero era externa a sus actividades habituales. En el pasado, la ciencia y la medicina estaban revestidas de un importante grado de mística; hoy, la relación entre la medicina y la población se construye alrededor de un proceso dialéctico entre una necesaria confianza y la duda, el respeto y la desilusión. Una confianza activa que ahora debe ser ganada y retenida, considerando una situación de incertidumbre creciente.

Otra característica emblemática de la modernidad tardía es la notoriedad de los sistemas de comunicación escritos, audiovisuales y electrónicos. Tal mediación de la experiencia es particularmente sensible en la medicina, reflejando una actitud ambivalente y polarizada. Por un lado, 
contribuye a la mistificación de la biomedicina, exaltando logros espectaculares y sensacionalistas sin mayor filtro y, por otro, fomenta y sirve de vitrina para la exhibición de desatinos, errores y denuncias de la acción de los médicos. La salud y la enfermedad constituyen materia predilecta de opiniones y convicciones del imaginario colectivo.

2. La percepción de la libertad profesional disminuida. Los médicos se verán obligados a proyectar su ejercicio profesional casi exclusivamente en el marco de organizaciones en las cuales detenten la calidad de funcionarios. En ellas prevalece una racionalidad que no es sólo técnica (biomédica) sino que económica (a menudo descalificada como «economicistas), que emerge por la imperiosa necesidad de frenar el incremento de los gastos y los costos de la atención de salud.

Ante los ojos de los médicos como actores sociales, han irrumpido principios y conceptos como el de productividad, racionalización, costo beneficio, rendimiento, estimados en principio como ajenos, y aún más, sentidos frecuentemente como alienantes. El paradigma de la administración ha entrado a competir con el paradigma médico profesional e incluso a sustituirlo, en algunos casos, como principio legitimador de la organización y la gestión de las instituciones de salud. El locus de control se desplaza a un sistema gerencial, en que el criterio clínico es sólo un componente de la fórmula. Por esto, los médicos sienten que su autonomía profesional, y por ende, su libertad, se ha visto limitada por motivos adicionales a la actitud de los pacientes.

«Cambios... la parte económica que nunca nos enseñaron a manejarla sino que la hacían otros, o sea la gestión. No se habla de lo económico, pero está siempre presente». (Médico, Grupo Focal I).

Otro recorte sobre la autonomía del médico se expresa en la instrumentación creciente de mecanismos de regulación y auditoría del rendimiento y desempeño del médico. El acento en la eficacia clínica, en la medicina basada en la evidencia y en la estandarización de protocolos y guías clínicas, es estimado por muchos médicos como un indicador de la presión creciente para controlar sus prácticas, apuntando a conocer qué, cómo y cuánto se diagnostica, se trata y se prescribe.

«a práctica grupal requiere de sistemas de administración que registren detalladamente nú- mero de casos atendidos y documentar detalladamente la eficacia de las terapias administradas... obliga a los profesionales a gastar tiempo y esfuerzo en la preparación de formularios e informar de sus acciones a los servicios de salud en el sistema público y a las compañías de seguros e ISAPRES en el privado, algo a lo que no están acostumbrados profesionales que han sido socializados con elevados grados de autonomía». (Médico, Encuesta I).

Tales apreciaciones podrían considerarse una herida en el corazón del concepto mismo de profesión, tal como lo describió Eliot Freidson ${ }^{3}$, quien defendió la tesis de que en la esencia de la profesión médica se encuentra la facultad de orientar y controlar el contenido del propio trabajo. La palabra profesión proviene del latín professio-onis, acción y efecto de profesar, aludiendo a la idea de dedicación y desinterés, ya que profesar implica no sólo ejercer un saber sino que también proclamar públicamente y comprometerse con una creencia ${ }^{4}$. Es por eso que el sacerdocio, la medicina, y el derecho fueron oficios considerados tradicionalmente profesiones en la mayoría de las sociedades occidentales. En términos generales, se ha definido a la profesión como una ocupación que monopoliza una serie de actividades sobre la base de un gran acervo de conocimiento abstracto que permite, a quien lo desempeña, una considerable libertad de acción, con importantes consecuencias sociales. El concepto ha estado ligado a la evolución y desarrollo de las sociedades; sin embargo, su significado actual se vincula con la industrialización y su consecuente división del trabajo. En efecto, las profesiones comienzan a separarse de la esfera religiosa en la modernidad, adoptando el sentido que hoy les damos, a partir de la reforma protestante.

Centrándose paradigmáticamente en la medicina, Friedson, a fines de la década de los setenta, marcó un hito en el estudio de las profesiones, las que, en contraste con otro tipo de ocupaciones, tendrían la capacidad para acumular y utilizar conocimiento bajo sus propios estándares, sin estar sujetas a control por actores externos ${ }^{5}$. El desarmollo de los acontecimientos ha revelado, sin embargo, las limitaciones del modelo para la comprensión de la realidad actual. Sin desconocer su valioso aporte analítico, los nuevos discursos expertos disponibles a partir de los ochenta, le otorgan mayor peso al entorno social en que se desenvuelve la profesión, reemplazando un paradigma fundamentalmente taxonómico o descrip- 
tivo de características para el análisis de las profesiones, por otro histórico. Se presta atención particularmente a las nuevas orientaciones del estado en salud, a los agentes privados encargados del financiamiento, la contratación y regulación de los costos y a la dinámica interacción entre éstos. En este entorno, el liderazgo médico debe ahora compartir el poder de decisiones con los administradores y gerentes y además con otros profesionales de la salud y con los propios pacientes. Tales modelos de regulación de las profesiones se sitúan en un marco político en el que se ha ido construyendo progresivamente un nuevo equilibrio de poder. En este marco es donde entendemos el llamado a construir un nuevo «contrato social»aludido en los trabajos recientes del British Medical Journal que podría contribuir a contrarrestar este fenómeno, sentido por los médicos como de «acorralamiento». Edwards y $\mathrm{Col}^{5}$, plantean que el descontento de los médicos se asocia a cambios en las expectativas de pacientes, gobiernos y empleadores, no explicado sólo por la carga laboral y la remuneración. El factor clave para comprender este fenómeno sería la existencia de un cambio de un orden mayor, en el pacto psicosocial que existía entre la profesión, los empleadores, los pacientes y la sociedad. Es decir, el trabajo es ahora diferente a lo que los médicos esperaban que fuera. Esta modificación ha ocurrido sin una discusión explícita entre los involucrados y sin instalar en su reemplazo una alternativa que sea igualmente significativa y gratificadora. En esta línea, Smith ${ }^{7}$ aboga por el reemplazo de un pacto fraudulento»por uno más realista, en el cual pacientes y médicos deben saber y acordar que la muerte, la enfermedad y el dolor son parte de la vida y que la medicina es riesgosa y posee un poder limitado, particularmente para solucionar problemas sociales.

«l público ha cambiado y siente derecho a exigir estar sano. La gente no quiere morirse y nos exigen que no se mueran... y la medicina ha cambiado en todo el mundo; entonces, cómo sustentar este deseo de la gente de no morir, no tener enfermedad». (Médico, Grupo Focal II).

4. La segmentación de la profesión y la crisis de la representatividad. La práctica médica hoy se realiza en ambientes organizacionales cada vez más estructurados y de alta complejidad tecnológica. La tecnología representa para algunos médicos una fuente de tensión y para otros constituye una oportunidad para mejorar la calidad de práctica, dependiendo de la edad, la especialidad y el sector de práctica.

«l arte es ahora reemplazado por la tecnología. Hay un abandono de la posibilidad de adquirir destrezas clínicas de un nivel importante porque se reemplazó por la tecnología. Esto hace que se abuse y se pidan más exámenes de los necesarios». (Médico, Grupo Focal II).

«.. (en mi especialidad) que partió con una tasa de mortalidad muy alta, la tecnología ha sido muy útil y por eso la satisfacción es grande». (Médico, Grupo Focal II).

« El desarrollo tecnológico de la medicina es impresionante. La medicina dio un salto enorme y es difícil adaptarse para los médicos de cierta edad. Aunque hoy es más fácil diagnosticar». (Médico, Grupo Focal III).

Asociada a la creciente innovación en el equipamiento tecnológico, la especialización, y frecuentemente la hiperespecialización, es una tendencia ya instalada. Esto, a su vez, ha reforzado la heterogeneidad dentro de la profesión, con lo cual parece cada vez menos legítimo, desde un punto de vista social, hablar de la medicina o de los médicos. Este fenómeno no es nuevo. A lo largo del siglo diecinueve y parte del veinte, por ejemplo, en muchos países occidentales la oposición de intereses entre médicos urbanos y médicos rurales fue una constante ${ }^{7}$. Pero parece que las brechas y contradicciones se han ampliado y profundizado.

Durante las últimas décadas la especialización ha jugado también un rol táctico para obtener un nicho dentro de un mercado laboral progresivamente más competitivo, en que el componente tecnológico le agrega valor económico al acto médico ${ }^{8}$.

Además del factor tecnológico y de la especialización, otra estrategia para la generación de mayores ingresos es la acumulación de varios empleos. El multiempleo médico, que en la mayonáa de los casos constituye una práctica física y psicológicamente agobiante, más que una situación comparativamente ventajosa, es muchas veces, el camino para lograr un monto de ingreso mensual razonable.

«eferente a calidad de vida... mucha carga laboral por escasos recursos, que implica muchas horas de trabajo y diversos lugares para hacer un sueldo». (Médico, Encuesta II).

En un entorno progresivamente más competitivo y percibido como hostil, las relaciones entre 
los pares cobran una relevancia crítica, pudiendo ser en algunos casos, fuente adicional de tensión, en otras instancias, valoradas como una fuente de apoyo emocional e instrumental e incluso añoradas como parte de un pasado más gratificante de una práctica médica de mayor prestigio social.

«Como que hay que achatar para que nadie brille. Los referentes son (extranjeros); uno no tiene guías porque todos están al mismo nivel, ese guía era el que enseñaba el arte... la desaparición de los grandes ma estros produce un deterioro en la relación con los pares». (Médico, Grupo Focal II).

«Acá es un grupo de apoyo. Este grupo humano es un refugio, humor, etc. Es tu segunda familia». (Médico, Grupo Focal III).

«no necesita el aplauso a la buena práctica. Yo vivía mucho más angustiado en el hospital (público), con más susto pero también con más satisfacción. El estar juntos tiene también ese reconocimiento entre los pares... mi nostalgia es otra, es la del par que te pone nota. Los becados, internos, uno hablaba para la galería». (Médico, Grupo Focal I).

La diversidad de clivajes o nichos profesionales entre los médicos ha dado origen a diferencias evidentes y también a otras más sutiles, culturales y de mentalidades, de expectativas e intereses, lo cual plantea una crisis en la representatividad grupal, ocupacional. La variedad de intereses se torna compleja de coordinar y de gestionar, como

\section{REFERENCIAS}

1. Wimams S, Calnan M. The limits' of Medicalization? Modern Medicine and the Lay Populace in 'Late' Modernity. Soc Sci and Med 1996; 42: 12: 1609-20.

2. Giddens A. Un mundo desbocado. Los efectos de la globalización en nuestras vidas. España, Editorial Taurus. 2000.

3. FREIDSON E. La profesión médica. Un estudio de sociología del conocimiento aplicado. Barcelona: Península, 1978.

4. Gómez VM, Tenti Fanfani E. Universidad y profesiones. Buenos Aires: Miño y Dávila Editores, 1989. se evidencia en el diálogo político con el estado y con otros actores de la sociedad civil, que ha podido observarse en nuestro país particularmente en torno a la reforma de la salud, y que es, por lo demás, un fenómeno internacional. Este hecho no ocurre exclusivamente en la medicina, sino que parece ser un rasgo común a las profesiones, en la medida en que sus integrantes comienzan a tener situaciones de trabajo (o desempleo) heterogéneas e incluso contrapuestas. Tienden a declinar, como consecuencia, los aparatos corporativos tradicionales de representación única, para dar paso a diferentes grupos de presión más específicos y de mayor representatividad.

Los procesos descritos no agotan el panorama actual de los cambios de mayor impacto en la profesión médica. Los temas seleccionados en este análisis corresponden a las preocupaciones prioritarias que emergieron de la conversación con los distintos grupos de médicos participantes en este estudio. Ellas han sido incorporadas en un análisis que ilustra el contexto estructural que contribuye a determinarlos. Quedan intocados otros procesos interesantes como, por ejemplo, el de la feminización de la medicina, la emengencia y notoriedad de la denominada medicina alternativa o complementaria, que forma parte del fenómeno cultural alternativo general, y las nuevas relaciones entre la medicina y las otras profesiones de la salud.

5. EdWARDS N, KoRnacki MJ, SiLversin J. Unhappy doctors: What are the causes and what can be done? BMJ 2002; 324: 835-8.

6. Sмттн R. Why are doctors so unhappy? There are probably many causes, some of them deep. Editorial. BJM 2001; 322: 1073-4.

7. CoBurn D. State Authority, Medical Dominance and Trends in the Regulation of the Health Professions: the Ontario Case. Soc Sci and Med 1993; 37: 841-50.

8. Katz J, Miranda E. Mercados de salud: morfología, comportamiento y regulación. Revista de la Cepal 1994; № 54 Diciembre: LC/G.1845-P. 